\title{
SCIENTIFIC ASTRONOMICAL SCHOOL BY PROFESSOR VOLODYMYR P. TSESEVICH ON THE PHYSICS OF VARIABLE STARS
}

\author{
I.B. Vavilova \\ Main Astronomical Observatory, National Academy of Sciences of Ukraine, \\ 27 Akademika Zabolotnogo, Kyiv, 03143, Kyiv, Ukraine \\ irivav@mao.kiev.ua
}

\begin{abstract}
This paper is dedicated to the Prof. Volodymyr Platonovych Tsesevich (1907-1983), an outstanding scientist and legendary personality of the XX century.

We describe briefly the Kyiv period of his life and activity taken from his Personal Dossier from the Archive of the Presidium of the NAS of Ukraine. A particular attention is paid to the role by V.P. Tsesevich in the development of astrophysical research at the Main Astronomical Observatory of the Academy of Sciences of UkrSSR, when he served as the Director (19.11.1948-03.05.1951), and to the fruitful cooperation between Kyiv and Odesa astronomers.
\end{abstract}

We present briefly a "tree" of the scientific astronomical school by Prof. V.P. Tsesevich on the physics of stars. The data were obtained from different archives (Astronomical Observatory of the I.I. Mechnikov National University of Odesa, Main Astronomical Observatory of the NAS of Ukraine, Archive of the Vernadsky National Library, Archive of the Russian AS, and other institutions). The full database contains of a brief information on the about 100 representatives of this school as follows: name, title and year of thesis's defense, past/present affiliation). The scientific school is formed since 1950-ies till now having its greatest continuation in the work of such astronomers as N.S.Komarov, V.G.Karetnikov, Yu.S. Romanov, and I.L.Andronov (a branch of this school after V.P. Tsesevich), as well as S.M.Andrievsky as the follower by V.G. Karetnikov and T.V. Mishenina, V.F. Gopka, V.V. Kovtykh as the followers by N.S. Komarov.

The given information on the school by V.P. Tsesevich is not absolutely full, for example, 1) there are no the data on thesis's defense under his supervision before 1948; 2) information on the astronomical school developed by A.M. Stafeev and some other scientists is a very poor; 3) some shortcomings may be presented. We will grateful for all the additions and corrections to update a tree of this scientific school, which played and plays a prominent role in the development of our knowledge on physics of stars.

Keywords: variable stars, scientific school, personalities: V.P. Tsesevich.

\section{Introduction}

Prof. Volodymyr Platonovych Tsesevich (11.11.1907, Kyiv - 28.10.1983, Odesa) was outstanding scientist in the fields of astrophysics and astronomical instrumentation of the XX century. His main studies from student years till the last days of a life were concerned with the physics of variable stars (observation and theory). Being a

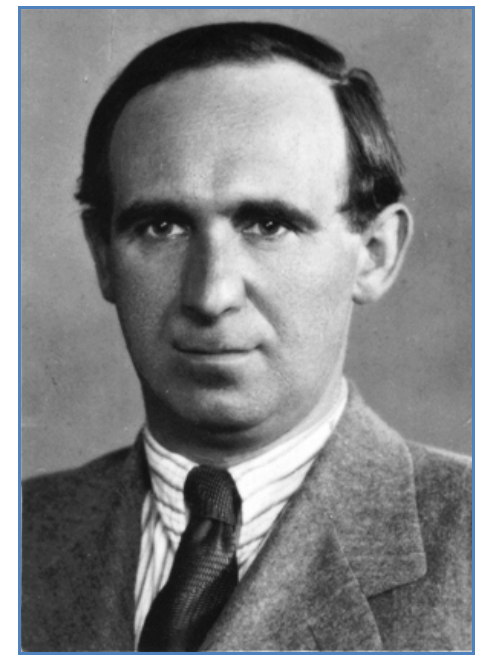

Figure 1: Professor V.P. Tsesevich, 1948.

Photo is taken from "The Personal Dossier of the Corresponding Member of the Academy of Sciences of UkrSSR. V.P. Tsesevich", Archive of the Presidium of the NAS of Ukraine

great enthusiast of astronomy, brilliant lecturer and popularizer, V.P. Tsesevich has engaged many young people and collaborators in this research and was able to create a world recognized scientific astronomical school of his own. He was a foremost organizer of science, for example, his activity in frame of the International Astronomical Union was as follows: Past Vice-President of Com. 42 Close Binary Stars (1961-1967), Past Organizing Committee Member of Commission 42 Close Binary Stars (1967-1973).

The biography of V.P. Tsesevich and his scientific work and activity as the Director of the Observatory in Dushanbe in 1933-1937 (now - Institute of Astrophysics of the Academy of Sciences of the Republic of Tajikistan) and the Director of the Astronomical Observatory of the I.I. Mechnikov National University of Odesa in 19441983 is fully described by his colleagues (see, for example, Karetnikov (1996), Pozigun (1996), Karetnikov (1997), Andronov (2003), Koshkin \& Mishenina (2007), Fenina \& Romanov (2008), Andronov (2017)).

By this reason, in this paper, we underline briefly the Kyiv period of his life and activity (Chapter 2), namely his role in the development of astrophysical research at the Main Astronomical Observatory of the Academy of Sciences of UkrSSR (MAO NASU), when he served as the Director (19.11.1948-03.05.1951) as well as was elected as the Corresponding Member of the Academy of Sciences of the UkrSSR in 1948 (Figure 1). 
During the 20th century in Ukraine several recognized astronomical centers were established and scientific school were developed thanks to the activity of such prominent astronomers as A.Ya. Orlov in Odesa, Poltava, Kyiv (see, Yatskiv et al., 2005); N.P. Barabashov in Kharkiv (see, Shkuratov, 2008); S.K. Vsekhsvyatsky in Kyiv (see, Andrienko \& Zosimovich, 2005; Vavilova et al., 2011); S.Ya. Braude in Kharkiv (see, Konovalenko, 2000; Vavilova et al., 2007); and V.P. Tsesevich in Odesa (see, references, in the previous paragraphs). The modern educational and scientific system of Ukraine in field of astronomy and astrophysics has been provided due to their extensive work (Pavlenko et al., 2006; Yatskiv et al., 2003). In this context, we present also briefly a "tree" of the scientific astronomical school by V.P. Tsesevich on the physics of variable stars (Figure 2, Chapter 3).

2. The Kyiv period of life and activity of Prof. V.P.Tsesevich

In 1944, V.P. Tsesevich was appointed to the position of professor of the Odesa University and Director of the Astronomical Observatory of this university. In 1944, December 30, he defended Dr. Sci. Thesis "On the methods of determining the orbits of Algol type stars" at the Kazan State University.

In 1948, Prof. V.P. Tsesevich was elected as the Corresponding Member of the Academy of Sciences of the UkrSSR (AS UkrSSR). His Personal Dossier in the Archive of the Presidium of the NAS of Ukraine contains of the characteristics and recommendation letters signed by Dr. Sh. Gordeladze (MAO AS UkrSSR, 11.05.1948), Dr. L. Shaposhnikova (Odesa State University, 20.05.1948), Prof. S. Orlov and Prof. B. Kukarkin (AstroSovet AS SSSR, 05.11.1946), Prof. D. Martynov (Kazan University, 23.05.1948), Prof. A. Mikhailov (AstroSovet AS SSSR, 23.05.1948). These letters give additional support to the recognition of V.P. Tsesevich's results in those years. We can read, for example: "In 1931, together with B. Okunev, he organized a regular observational service for RR Lyratype stars, which continues to the present day. Studying the Blazhko effect, he established the relationship between the nature of changes in their periods and the spatialkinematic characteristics. Tsesevich V.P. conducted a series of studies of stars such as RV Tauri, cepheids (periods, light curves), eclipsing variable stars. He improved the methods of determining the elements of orbits and other characteristics of eclipsing stars by their light curves (method of differential corrections), developed a method for taking into account the annularity of eclipses. In 19391940 he published tables of special functions for solving light curves for various types of eclipses, which till now are considered the best and unsurpassed in accuracy...".

A great support in this election was played by A.Ya. Orlov, Member of the AS UkrSSR, Director-Founder of the Main Astronomical Observatory of the AS UkrSSR. He invited Volodymyr Platonovych to Kyiv to replace him for the post of Director.

In 1948-1951, Prof. V.P. Tsesevich headed (part-time) MAO AS UkrSSR, being at the same time the Director of the Astronomical Observatory of the Odesa University
(OAO). There are no documents, which shed light what has caused his decision. But years later, one can say that this decision was the only true and wise one. Although V.P. Tsesevich was the Director for only three years, he significantly influenced the development of astrophysical research as well as initiated a number of scientific and organizational reformations at the Main Astronomical Observatory of the AS UkrSSR:

- suggested new research fields: Photographic Astronomy (sky survey); Variable Stars; Structure of the Galaxy;

- organized the Scientific Council of the Main Astronomical Observatory: the first meeting was held in 1949;

- established the journal "Izvestiya GAO AN UkrSSR" ("News of the MAO AS UkrSSR"): the first issue was published in 1950;

- held the IX Plenum of the Commission for Research on Variable Stars of the AstroSovet of the Academy of Sciences of the USSR in 1950.

- invited the students graduated from the Odesa University, Department of Astronomy, to begin their scientific career and research at the Main Astronomical Observatory in Kyiv: the first student, who has responded to this initiative, was Raisa Chupryna. She worked at the observatory in 1949-1957 and afterwards at the Odessa Department of the MAO NASU.

- initiated postgraduate courses for future Candidates of Science (PhDs) in astrophysics at the Faculty of Physics of the Kyiv University: the first Candidates of Sciences, who defended their theses under V.P. Tsesevich's supervision and began to form a Kyiv's branch of his scientific astronomical school, were I.G. Zhdanova and F.I. Lukatskaya in 1953 (see, Figure 2).

Later on, the "Odessa landing" of post-graduated students has arrived to the MAO NASU. Some of them defended their theses under Tsesevich's supervision as well as have formed the department of physics of stars: M.Ya. Orlov (research of RCrB type stars with anomalous chemical composition, worked in 1960-1998), T.V. Orlova (research in solar physics, worked in 1960-1985), L.R. Lisina (photometric research of the Moon, worked in 1960-1994), M.G. Rodriges (research of stars of late spectral classes, worked in 1960-2002), A.F. Pugach (research of physics of non-stationary stars, worked in 1962-2011), E.S. Kheilo (variable stars in globular clusters, astronomical instrumentation).

The new round of cooperation between observatories fell on 1972-1983 years and related to the initiatives by Ya.S. Yatskiv and V.P. Tsesevich:

- in 1972, the Odessa Department of Astrophysical Instrumentation of the MAO NASU ("Zeiss-Sevich" firm) was established; the telescope (diameter $0.8 \mathrm{~m}$, Cassegren's focus $\mathrm{F}=10 \mathrm{~m}$ ) of the Astronomical Observatory of the Odesa University was installed at the Peak Terskol (Kabardino-Balkaria, Russia). Prof. V.P. Tsesevich was the Head of this department on a voluntary basis;

- in 1978, the Bureau of the Presidium of the Academy of Sciences of the UkrSSR decided to create a network of decameter radio telescopes "URAN", including telescope "URAN-4" in Mayaki, near Odesa, where the OAO Observational Station is located. Now this antenna belongs to the Institute of Radio Astronomy of the NAS of Ukraine. 

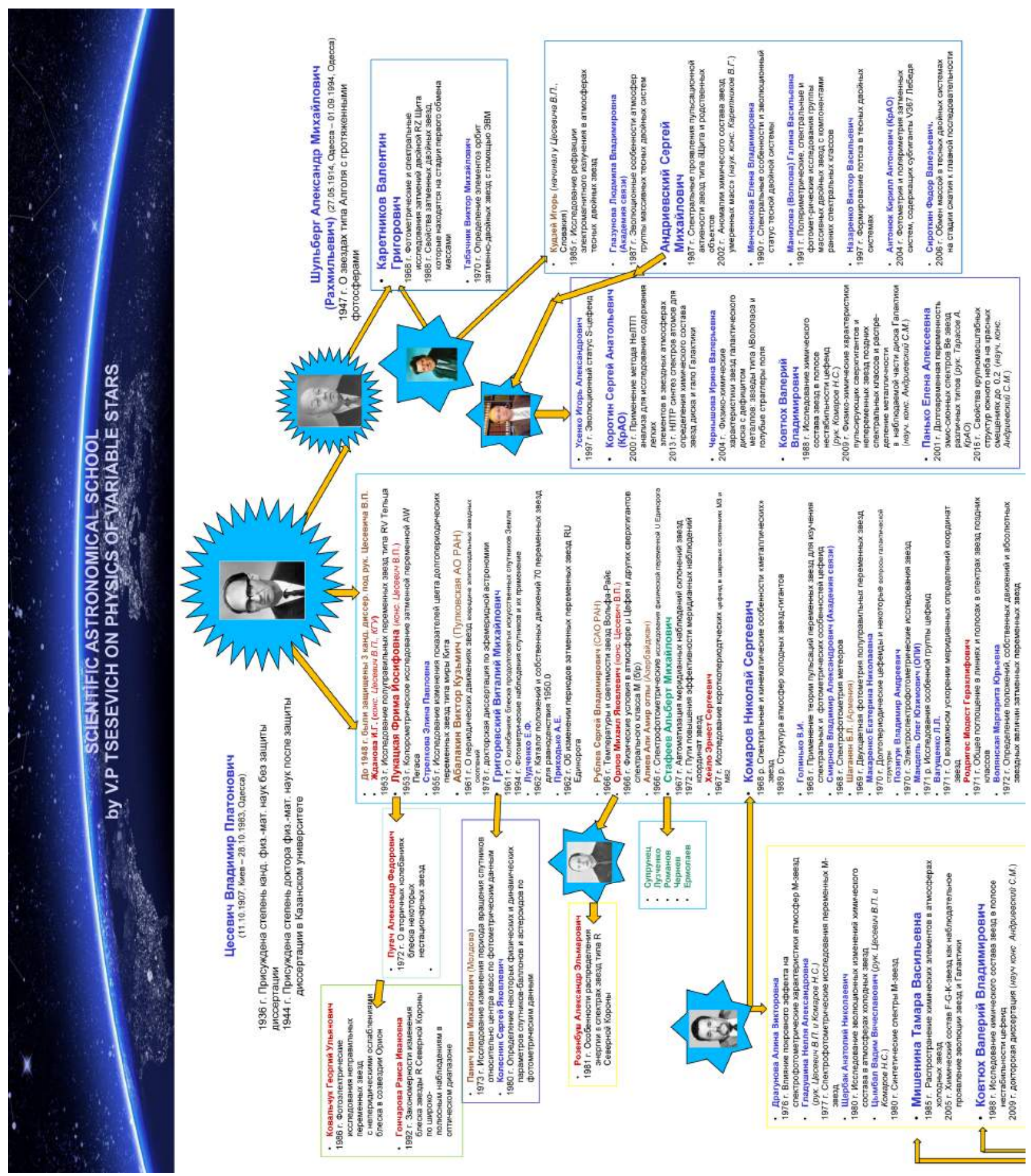

Figure 2: The tree of the scientific astronomical school by Professor V.P. Tsesevich on variable stars.

This poster (in Russian) was presented during the Gamow Conference-School in Odesa, August 14-19, 2017, at the Memorial Session dedicated to the 110-anniversary of Prof. V.P. Tsesevich' birthday.

Since then it was slightly changed after the helpful remarks by Prof. V.G. Karetnikov. 

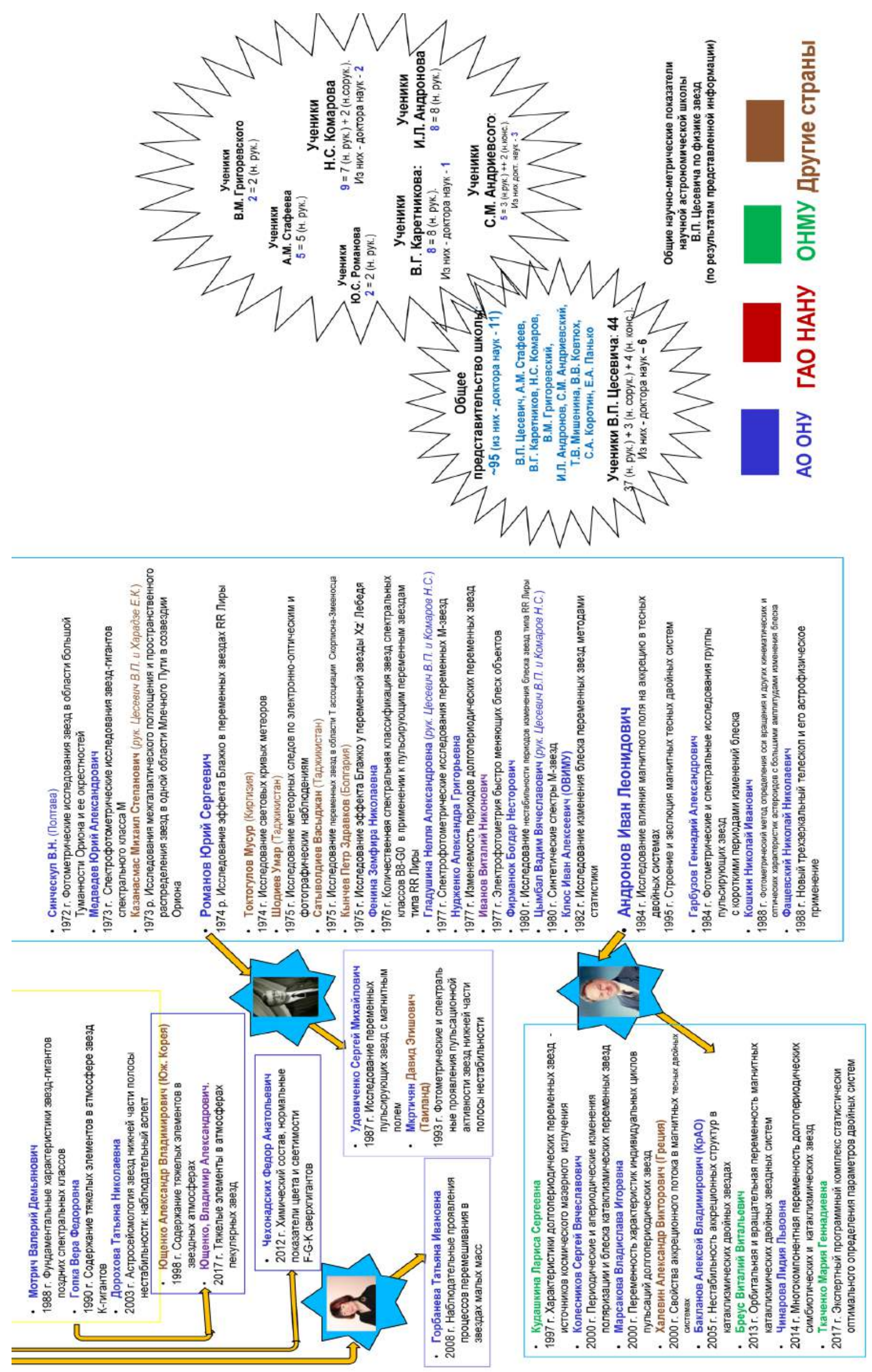
This cooperation continues to this day in frame of such projects as follows:

- Ukraine Network of Optical Stations (UMOS) for near-Earth space research, responsible persons for these observations from the $\mathrm{OAO}$ are N.I. Koshkin and V.V. Troyansky (http://umos.mao.kiev.ua/);

- Ukrainian Virtual Observatory (UkrVO), responsible person for this research field from the OAO is S.V. Kashuba (http://ukr-vo.org), see, for example, Vavilova et al. (2013);

- "CosmoMicroPhysics", Target Complex Scientific Program of the NAS of Ukraine, which was conducted in 2017-2013; responsible person for this research from the OAO was Prof. A.I. Zhuk. It is concerned with a study of the structure and evolution of the Universe on galactic and cosmological scales, dark energy and dark matter problem (see, for example, Novosyadlyj et al., 2013; Yatskiv et al., 2014; Alexandrov et al., 2016);

- joint research in field of physics of stars (see, for example, Gopka et al. (2013));

- participation in the Gamow conferences-schools, including membership in the organizing committee.

In 2017, a newest round of cooperation obtained a legal confirmation: "The Odesa Scientific \& Educational Complex" was established by the Astronomical Observatory of the I.I. Mechnikov National University of Odesa and the Main Astronomical Observatory of the NAS of Ukraine. It was initiated by Prof. Ya.S. Yatskiv and Prof. S.M. Andrievsky and was supported by Prof. I.M. Koval', the Rector of this University.

\section{Scientific Astronomical School by V.P. Tsesevich on variable stars}

A scientific school is one of the forms of organization of scientific research, which is extremely productive for the development of science, especially in the so-called scientific growth points. This form of organization of scientific activity presupposes availability:

- the presence of an informal structured scientific or engineering community;

- the ability of this informally structured community to offer a new field in research, create new knowledge, formulate new ideas and concepts, solve a number of problems at the global avant-garde level, that is, the ability to create a high science;

- the ability of this informally structured community to create itself or use high-tech equipment and knowledge from related scientific fields or industries;

- the founder of the scientific school, leader-scientist/ the design engineer, who for the first time forvarded a new problem or a new field, developed it himself and with his students and followers;

- a special style of work and thinking, a special atmosphere, a respect for the students and the opportunity for them to work together, a pedagogical work on selecting students and improving their knowledge, that is all that lays the foundations of continuity of the school.

Scientific school is an organism with its life cycle, but this cycle may be limited. The final of a scientific school is due to various factors, in particular: the educational resources have been exhausted; innovations have been lost; there have been revolutionary changes in the scientific field and the scientific school does not have time to reorganize own research, and so on. As a result, the school can "dissipate", turn into smaller research groups and laboratories that keep traditions, and maybe give birth to new scientific idea. We note that scientific schools have a greater level of professionalism and experience to reach scientific results as compare with level of mobility and ability to rapid changes in methods of investigation. At the same time, the growth of experience prevents mobility, and the growth of mobility leads to loss of experience.

The scientific astronomical school by Prof. V.P. Tsesevich on physics of variable stars meets the aforementioned definitions of scientific school. Its tree is presented in Figure 2. The data were obtained from various archives (Astronomical Observatory of the I.I. Mechnikov National University of Odesa, MAO NASU, Archive of the Vernadsky National Library, Archive of the Russian AS, and other institutions). The full database contains of brief information on the about 100 representatives of this school as follows: name, title and year of thesis's defense, past/ present affiliation).

The scientific school has been formed since 1950-ies and had a greatest continuation in the work of such astronomers as V.G. Karetnikov, N.S. Komarov, Yu.S. Romanov, and I.L.Andronov (a branch of this school after V.P. Tsesevich), as well as S.M. Andrievsky as the follower by V.G. Karetnikov, and T.V. Mishenina, V.F. Gopka, V.V. Kovtykh as the followers by N.S. Komarov.

The research fields, where V.P. Tsesevich, his postgraduate students and followers make foremost results, could be compiled (in period till 1980-ies) as follows:

- physics of variable stars of different type (epoch 1 before observations with the $7^{\text {th }}$ camera astrograph, and epoch 2 - after its installation at the observational station in Mayaki);

- systematical monitoring and photography of the sky with the help of multi-camera short-focus astrographs, which was provided in Ukraine and Tajikistan;

- catalogues and atlases of variable stars and other cellestial bodies;

- Blazhko effect testing for different type of variables;

- definition of the orbits of binary stars with extended atmospheres (A.M. Shulberg and his followers);

- astronomical ground-based instrumentation.

The most of results of this period were integrated in monographs and books (Tsesevich, 1969; Tsesevich \& Kazanasmas, 1971; Tsesevich, 1971; Tsesevich, 1973; Tsesevich, 1976). A full biobibliography by V.P. Tsesevich was issued by Dziubina \& Rikun (1988).

New investigations, which were initiated in 1957 under the program of the International Geophysical Year since the beginning of space research, are provided till now:

- Sky Patrol, studies of changes in the brightness of the artificial satellites of the Earth (see, for example, Grigorevskii, 1979; Koshkin, 2017);

- observations of meteors (see, for example, Gorbanev \& Kramer, 1994); we note that V.P. Tsesevich initiated together with E.N. Kramer the All-Union Fireball Service.

Since 1980-ies his followers have formulated new ideas and concepts and strengthened the success and international cooperation of the scientific school at a higher level: 
- photometrical and spectroscopic study of variables (see, for example, Karetnikov, 1986; Cherepashuk, 2003);

- astrospectroscopy (see, for example, Komarov, 1985; Gopka, 2004; Yushchenko, 2002; Mishenina, 2016; Andrievsky, 2018; Kovtyukh, 2006; Korotin, 2015);

- astroseismology (see, for example, Mkrtichian, 2017);

- "Inter-Longitude Astronomy" (Andronov, 2003);

- and other research fields, which are integrated with scientists who is not belonging to this scientific school (extragalaxy astronomy, cosmology etc.).

The international campaign "Inter-Longitude Astronomy" collects smaller campaigns on different objects of numerous types - "magnetic" and "non-magnetic" cataclysmic variables, classical eclipsing and interacting binary systems, pulsating and eruptive variable stars. Review on the theoretical models vs observational evidence of gravi-magnetic rotators (classical, asynchronous and intermediate polars) and on the methods for statistically optimal phenomenological modelling using "special shapes" are given by Andronov (2008, 2016).

At the end of this Section we would like to give several commentaries. First of all, about the greatest collections, which are at the disposal of the OAO: 1) the glass collection that ranks the third in the world after Harvard and Sonneberg Observatories and numbers 105000 photoplates. This collection was formed due to the efforts of astronomers of Odesa during long-term observations mainly with $7^{\text {th }}$ camera astrograph in Mayaki; 2) the unique part of glass collection, which is stored in Mayaki, is the so-called Simeiz collection of astronegatives. It contains of the observational data (since 1909) on small bodies of the Solar System, which were obtained at the Simeiz Observatory in Crimea. This collection was saved by V.P. Tsesevich and moved to Odessa after he accidentally found it discarded at the Crimean Astrophysical Observatory, - a great respect to Prof. Tsesevich for this decision.

A commentary to the tree of scientific school is related to the personalities and questions, which are raised when you consider abstracts of their Cand. Sci. Theses. The abstracts of theses until the 1960's have consisted of several pages and did not always contain information on the name of the scientific supervisor. In such cases, one who is a supervisor has to judge from the subject of the dissertation, from the comments of colleagues, as well as from own knowledge of the history of astronomy in Ukraine.

A very important argument in the compilation of a science school's tree is the discussion with representatives of the school, as it gives an opportunity to better understand the role of each of them in the formation of the school, international cooperation, the ideas and methods proposed, etc. One example of the necessity of such approach is the candidate's dissertation by V.K. Abalakin, the outstanding astrophysicist of the XX century, one of the best graduates of the Odessa University, member of the Russian Academy of Sciences. The supervisor of his postgraduate courses and Cand. Sci. Thesis was V.P. Tsesevich, but the actual supervisor and the personality, who inspired Abalakin by celestial mechanics, was Professor K.N. Savchenko, of which Viktor K. Abalakin himself wrote brilliantly in his essay (Abalakin, 1995).

So, one can analyze scientometric indicators, which are given in Figure 2.
We can conclude that the age of the scientific school by V.P. Tsesevich is about 70 years; the averaged effectiveness of defense of Cand. Sci. Theses is 6 persons per 5 years; 11 persons among 95 representatives of this school defended Dr. Sci. Theses and 8 of them were supervisors at least 1 Cand. Sci. The Scientific School is living!

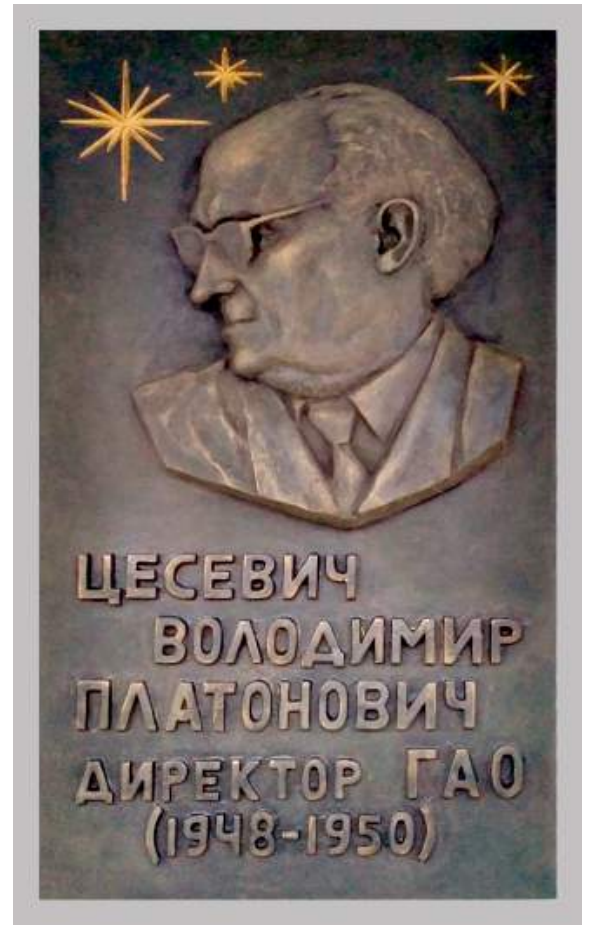

Figure 3: Memorial plaque in honor of V.P. Tsesevich, which was installed at the building of the MAO NAS of Ukraine on October 25, 2017. Sculptor - A. Kusnetsov

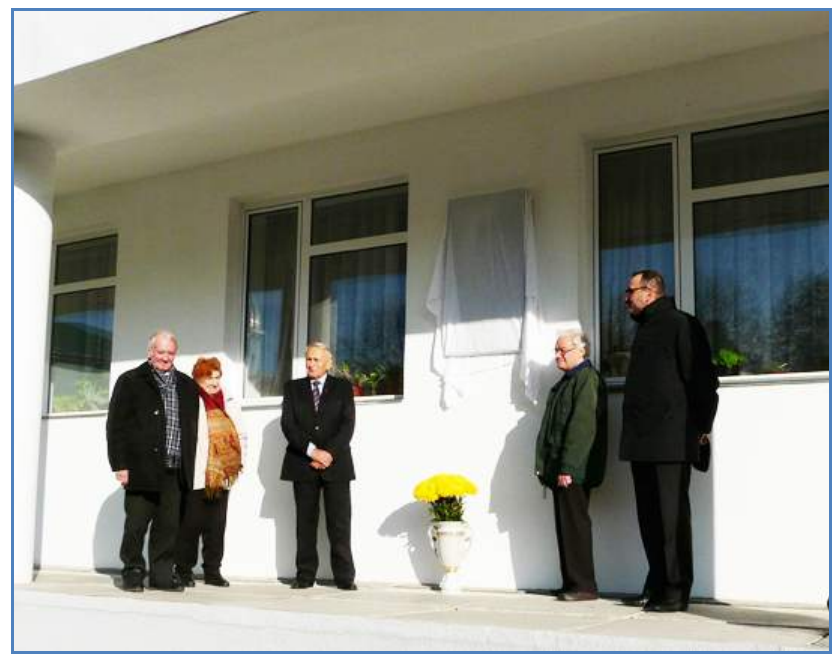

Figure 4: Ceremonial of the opening of the Memorial plaque in honor of V.P. Tsesevich, which was installed at the building of the MAO NAS of Ukraine. From left to right: Dr. M.I. Ryabov, Dr. A.A. Korsun', Prof. Ya.S. Yatskiv, Dr. M.G. Rodriges, Prof. M.V. Strikha (DeputyMinister for Education and Science of Ukraine). Kyiv, October 25, 2017.

We note that the given information on the scientific school by V.P. Tsesevich (Figure 2) is not absolutely full, 
for example, 1) there are no the data on thesis's defense under his supervision before 1948; 2) information on the astronomical school developed by A.M. Stafeev or scientists from other countries is a very poor; 3) some shortcomings may be presented. We will grateful for all the additions and corrections to update a tree of this scientific school, which played and plays a prominent role in the development of our knowledge on physics of stars.

\section{Instead of Conclusion}

On 25 October, 2017, a special session dedicated to the 110-anniversary of the Volodymyr Tsesevich birthday was organized in Kyiv in frame of the meeting of the Ukrainian astronomer named after "AstroAutumn in Holosiyiv".

Ya.Yatskiv, I. Vavilova and A. Korsun' have presented the report "Volodymyr Tsesevich and his activity as the Director of the Main Astronomical Observatory of Academy of Science of the UkrSSR". Mikhail Ryabov has told his impression on the V. Tsesevich's role for development of astronomical instrumentation "30 years of radio astronomical research at the Odessa Observatory URAN-4" (see, for example, Ryabov, 1993).

Afterwards the participants from Chernivtsi, Dnipro, Kharkiv, Kyiv, Mykolaiv, Odesa took part in the opening the Memorial plaque in honor of Tsesevich V.P., which was installed at the building of the MAO NAS of Ukraine (Figure 3 and 4). The photo gallery is available through http://mao.kiev.ua/index.php/ua/?option=com content\&vi $\underline{\text { ew }=\text { article\&id }=270 .}$.

Acknowledgements. The author thanks Professors Ya.S. Yatskiv, V.G. Karetnikov and I.L. Andronov for useful remarks and Vavilov S.S. for technical assistance.

\section{References}

Abalakin V.K.: 1995, Professor K.N. Savchenko. In: “The Pages of the History of Astronomy in Odessa", Part 2, Ed. V.G. Karetnikov, Odessa: Astroprint, p.33-39.

Alexandrov A.N. et al.: 2016, General Relativity Theory: Recognition through Time, Kyiv: Naukova Dumka, 332 p.

Andrienko D.A., Zosimovich I.D.: 2005, in "Sergei Vsekhsvyatsky", Kyiv: Kyiv University, 8-38.

Andrievsky S. et al.: 2018, MNRAS, 473, Is. 3, 3377.

Andronov I.L.: 2003, Odessa Astron. Publ., 16, 5.

Andronov I.L.: 2008, JPhSt, 12, 2902.

Andronov I.L. et al.: 2003, $A \& A T, 22,793$.

Andronov I.L., Tkachenko M.G., Chinarova L.L.: 2016, PhysJ, 2, 140.

Andronov I.L.: 2017, Odessa Astron. Publ., 30, 252.

Cherepashchuk A.M., Karetnikov V.G.: 2003, ARep., 47, Is. 1,38

Dziubina S.V., Rikun I.E. (Comp.): 1988, Vladimir Platonovich Tsesevich. Biobibliography, Ed. Yu.S. Romanov, in "Scientists of Odessa", is. 17, $110 \mathrm{p}$.

Fenina Z.N., Romanov Yu.S.: 2008, Vladimir Platonovich Tsesevich or the Golden Age of the Odessa Astronomical Observatory. Kiev: LOGOS.

Gopka V.F. et al.: 2004, ARep., 48, Is. 7, 577.

Gopka V. et al.: 2013, Odessa Astron. Publ., 26/1, 54.

Gorbanev Yu.M., Kramer E.N.: 1994, Solar System Research, 27, No. 4, 376.
Grigorevskii V.M.: 1979, Pisma AZh, 5, 482.

Karetnikov V.G., Kovtyukh V.V.: 1986, AZh, 63, 1144.

Karetnikov V.G.: 1996, A\&AT, 10, 21.

Karetnikov V.G. (Ed.): 1997, The Pages of the History of Astronomy in Odessa. Collection, Part 4, Odessa: Astroprint, $208 \mathrm{p}$.

Komarov N.S.: 1985, AZh, 62, 740.

Konovalenko A.A.: 2000, Geophys. monograph, 119, 311.

Korotin S.A. et al.: 2015, $A \& A$, 581, id.A70, 10 pp.

Koshkin N.I., Mishenina T.V. (Eds.): 2007, "Vladimir Platonovich Tsesevich. About time and me". Reminiscences and documents, Odessa: Astroprint, $80 \mathrm{p}$.

Koshkin N. et al.: 2017, $A d v$. Sp. Res., 60, Is.7, 1389.

Kovtyukh V.V.: 2006, ARep., 50, Is. 2, 134.

Mishenina T. et al.: 2016, MNRAS, 462, Is. 2, 1563.

Mkrtichian D.E.: 2017, IBVS, No. 6210,\#1.

Novosyadlyj B. et al.: 2013, Dark Energy: Observational Evidence and Theoretical Models, Kyiv: Nauk. Dumka.

Pavlenko Ya.V., Vavilova I.B., Kostiuk T.: 2006, In: Organizations and Strategies in Astronomy, Vol. 7, Ed. A. Heck (Berlin: Springer), p. 71.

Pozigun V.A.: 1996, "Astronomical Observatory of the Odessa State University”, Odessa: Astroprint, 32 p.

Ryabov M. I., Serokurova N. G.: 1993, $A \& A T$, 4, Is. 1, 29

Shkuratov Yu.G. (Ed.): 2008, "200 years of astronomy in the Kharkov University", Kharkov: Kharkov National University, $632 \mathrm{p}$.

Tsesevich V.P.: 1980, Activities of A. Ya. Orlov in Odessa. In: Geodynamics and astrometry. Foundations, methods, results, p. 24-27.

Tsesevich V.P.: 1969, RR Lyrae stars (Jerusalem: Israel Program for Scientific Translations (IPST))

Tsesevich V.P.; Kazanasmas M.S.: 1971, Atlas of finding charts of variable stars, Moskva: Nauka, $350 \mathrm{p}$.

Tsesevich V.P.: 1971, Instationary stars and methods of their investigation. Eclipsing variables, Moskva: Nau$\mathrm{ka}, 352 \mathrm{p}$.

Tsesevich V.P.: 1973, Eclipsing variable stars, New York: J.Wiley.

Tsesevich V.P.: 1976, Investigation of variable stars in selected regions of the Milky Way, Kiev: Nauk. dumka, $256 \mathrm{p}$.

Vavilova I.B., Karetnikov V.G., Konovalenko A.A. et al.: 2001, In: "Preserving the Astronomical Sky", Eds. R.I. Cohen, W.T. Sullivan III, Proc. IAU Symp. 196, 153.

Vavilova I.B. \& Yatskiv Ya.S.: 2003, Teaching of Astronomy in Asian-Pacific Region, 19, 47.

Vavilova I.B., Konovalenko A.A., and Megn A.V.: 2007, Astron. Nachr., 328, 420.

Vavilova I.B. et al.: 2012, Kinemat. Physics Cel. Bodies, $\mathbf{2 8}$, is. 2,85 .

Shkuratov Yu.G. (Ed.): 2008, "200 years of astronomy in the Kharkov University", Kharkov: Kharkov National University, $632 \mathrm{p}$.

Yatskiv Ya.S., Vavilova I.B.: 2003, Kinemat. Physics Cel. Bodies, 19, 569.

Yatskiv Ya.S., Korsun A.A. \& Vavilova I.B.: 2005, Kinemat. Physics Cel. Bodies, 21, 403.

Yatskiv Ya.S. et al.: 2014, General Relativity: Horizons for Tests, Kyiv, MAO NASU, 264 p.

Yushchenko, A.: 2002, Journal of the Korean Astronomical Society, 35, 4, 209. 